\section{OBSERVATIONS ON CERTAIN FORMS OF DISEASE OF THE LUNGS.}

By Edward Lathair Oruerod, M.D.Cantab., F.R.C.P., Physician to the Sussex County Hospital.

III.-Phthisis : General Remarks; Grounds of Prognosis; Distinction hetween General and Circumscribed Tubercular Deposit; Specific Forms of the Disease; Acute Phthisis; Vomiring in early Phthisis; Arrested Phthisis; Regular Hectic ; Tussis Ferina; Persistent Cavities; Inflamiation of the Walls; Inlustrations of non-tubercular Cavities; Earthy Concretions ensuing on Degeneration of Tunercle; Arrest o Pithisis dy Colial'se of the Ling; General RecaPITUIATION.

AGaINst the belief which is daily gathering strength amongst us, that pulmonary consumption is not wholly beyond the reach of medicine, there are no insuperable objections, either on abstract or on particular trounds. When we claim a certain amount of influence for our treatment in arresting the progress of the disease, we claim no more than what the most impartial examination of the facts would allow; for, while morbid anatomy leads us directly to infer that tubercle is not incurable, by showing the most unequivocal evidences of tubercle in an obsolescent or quiet state in the apices of the lungs, clinical observation, with slowly accumulating wejght, is connecting these traces of past disease with the signs and symptoms of present phthisis. It is not enough to object generally to these observations the fallacy of confusion between simple inflammation and tubercular deposit; for it would be hard to name a subject in the whole range of practical medicine, which has been worked out with more jealous truth and accuracy of detail than the diagnosis of early phthisis, especially in reference to the curability of the disease. Few have this objection more constuntly in mind than the observers themselves.

Further: all that general observation concurs in as to the nature of phthisis, all that it approves as to the principles of treatment, and all that anatomy tells us of the possibly favourable results, is in strict accordance with what we might, it priori, infer from the elementary structure and mode of development of tubercle. 'The insight which the microscope gives us into the pathology of phthisis is indeed very obscure. Still, it says distinctly that the opinion of the curability of tubercle of the lungs is not unwarrantable.

The prospect, however, is less encouraging when we turn from abstract possibilities and general conclusions to the examination of particular cases. In the majority of cases, practically speaking, it is not as to the ultimate result, but as to how long that result may be delayed, that there is room for doubt and for hope. Generally, it may be said, that the probable duration of life in phthisis is inversely as the amount of the manifestation of disease in the lungs. But this rule has numerous exceptions; and least of all, at the commencement of the disease, is it of useful application. In fact, just at the time when the question is most important, it fails us: just when the alternative lies between days and years, instead of between one or two months-between the conduct of a life, may be, and the disposal of a few days-just then the rule is of no good. On this condition I would offer a few remarks.

I am not speaking of cases where hectic and other symptoms tell us that the pulmonary disease has struck deep root; but of cases where the ausence of these constitutional symptoms allows us to hope for a favourable result. Some mere chance, may be, has revealed the physical signs of disease; and the direction of some important step in life depends on their interpretation. This is no imaginary case : the question often takes a most practical form. 'The probable result of slight present indications of consumptive disease, and the prognosis in cases of early phthisis, are questions often asked in connexion with the marriage of young women of consumptive families. Is it safe or advisable for a young woman of a consumptive family, with any present unsoundness of the lungs, seemingly referrible to tubercular disease, to marry? is a question very commonly asked. Few would hesitate to answer that it is not safe; for, whatever other knowledge we may possess of the case, there are conditions introduced into the problem, of the particular application of which nothing is yet known, and on whose generally injurious influence all are agreed-pregnancy and subsequent lactation. Yet a contrary answer was correctly given in a case which I will briefly detail.

CASE I. Tubercle limited to the Apex of one Lung. In the spring of 1853 , I was consulted on the case of a lady 23 years of age, who had suffered from cough for two months. She had, besides, pain in the right side of the chest, and a feeling of irritation about the top of the sternum. She had occasionally suffered slight hæmoptysis. On auscultation, there were long expiration and pectoriloquy, with distinctly diminished resonance on percussion beneath the right clavicle.

I had several opportunities of repeating my examination, and assuring myself of the correctness of this observation. Under ordinary treatment, the cough soon subsided; but for some time there was an occasional slight spitting of blood.

She was engaged shortly to be married. An eminent physician, who had long known the family, was consulted, and gave an opinion, contrary to my own, that it was safe. She was accordingly married; and the sequel has hitherto proved the correctness of his opinion. She has been married upwards of four years, has borne two children, and, when I casually saw her in the autumn of 1858 , she looked in better health than ever I had seen her. But I had no opportuninity of examining her chest.

Yet she was delicate, and, I believe, of a delicate family; and, as far as I may build upon the auscultation, I should say that she had some small tubercular deposit, probably eroded in parts, in the apex of the right lung.

A few lines may suffice to reproduce the details of another case, which affords a striking contrast to this. I need scarcely say that the question of intended marriage arising in both these cases was wholly extraneous to the subject under discus. sion, except so far as it may have called attention to symptoms which might otherwise have been overlooked.

CASE II. Tubercle diffused through both Lungs. A lady, about 23 years of age, tall and healthy looking, but of a phthisical family, had enjoyed very good health, though she had rather overtaxed it by going out into society, till two months before I saw her. Then she had slight hæmoptysis. Since then, her health had been delicate, and she had more or less cough. On auscultation, the respiratory murmur was feeble all over the chest, perhaps more so on the right side than elsewhere. There was good resonance on percussion everywhere, except perhaps over the right scapular region, which was duller than the left

I spoke, as the very doubtful nature of the case indicated, in great uncertainty as to the result, but agreed in the expediency of deferring her marriage for the present.

In the course of a fortnight, some of the doubts had cleared up, unfavourably. She had suffered two or three attacks of rather free hæmoptysis; one, indeed, at the time of my visit, which prevented me from adding anything to the results already obtained, beyond recognising dulness on percussion beneath the right clavicle.

Three months afterwards, I saw her again. There was no doubt now. There was large crepitation all over the upper part of the right lung, before and behind; dulness on percussion to the same extent; enormous puriform expectoration; cough; and night sweats. In another three weeks, she was dead.

Yet I much doubted, on my first visit, that she had phthisis at all.

Now, taking these two cases as types respectively of two large classes of cases, I would draw an important pathological distinction between them. They represent very characteristically two distinct forms under which phthisis may present itself. In one of these forms, the tubercular deposit is circumscribed, and within those limits tends, by a certain series of changes, to obsolescence; in the other, the disease is diffused throughout the lungs, and tends to a rapid succession of the ordinary changes of tubercle. I think that, on closer examination, the pathological distinction between these two forms will appear to be real and important.

Of the more ordinary form of rapid phthisis, which the second case illustrates, there is no occasion to speak here; for of its terrible reality and importance there is no room to doubt. But, as to the former : first, are we justified in the belief that there is such a form of disease, limiting itself in such a way? And, secondly, assuming this, can we accurately ascertain, by physical or other signs, the existence of this particular form during life?

Morbid anatomy does not leave the affirmative answer of the first of these two questions a mere assumption. There is undoubtedly such a form: chalky degeneration of tubercle, with loss of substance of the lung, may very frequently be 
found limited to the apices of otherwise healthy lungs. But, secondly, can such a condition be detected by any means during life? For the most part, it is not. At least, the morbid anatomy of old tubercular deposit in the lungs, like that of old valvular disease of the heart, wants the histories to connect these organic changes with symptoms observed in the same individuals years before. But the question, whether it can be detected or not, is a different one. This question is, in other words, Is tubercular disease of the lung, thus limited, ever arrested, spontaneously or otherwise, after the disorganising changes have gone so far as to be cognisable by the ear?

As to the general possibility of the arrest of tubercular dis ease of the lung, at whatever period of its disorganising progress, clinical observation explicitly asserts the fact. (See, particularly, Bennett on Pulmonary Tuberculosis ; and Dr. R. Quain's Reports, Lancet, vol. i, 1852.) What may be the amount of organic disease which leaves a little puckering of the apices of the lungs, with a chalky concretion or two as its sole results, I cannot say; nor yet with what degree of accuracy its existence may be predicted from the physioal signs. But that some cases where the physical signs of phthisis had been observed get seemingly well, and that puckering and chalky concretions have been found, after death from other causes, to occupy the spots where those signs had been observed during life, and that the most frequent seat of thes favourable changes is the apex of the lung, rests on unquestionable evidence.

As to the particular application of this general law, there is more room for doubt. I think, however, that the physical signs of phthisis, thus limited, have a different and more hopeful import than under other circumstances; and that, in the absence of constitutional symptoms, the apparent limitation of the disease outweighs, as a favourable prognostic, the unfavourable inferences from a greater amount and a more advanced stage of the disease within those limits.

Often phthisis sets in almost imperceptibly, and creeps on slowly, perceived least of all by the patient himself ; and the disease is already far advanced when it first comes under our observation. It is sufficient here to say of such cases, that they commonly obey the general rule of succession of changes of tubercle of the lungs; and that the present amount and degree of advancement of the disease give a fair criterion of the probable duration of life in the particular case. But sometimes phthisis sets in under cover of acute disease, or its own proper symptoms assume that severity which draws attention to them at the outset. Cases of this kind have much interest, from the fact of our being able to assign with tolerable accuracy a date to the commencement of the disease. In the two cases already detailed, it was an accidental circumstance which caused the patients to attend to symptoms which they would otherwise have neglected. In the two next following, the symptoms themselves were urgent enough to bring the patients under observation. And here, again, I have placed the diffused and the circurnscribed forms of tubercular deposit in the strongest contrast.

Case IIr. Definite Outbreak of Tubercle limited to the Apex of one Lung. A delicate excitable girl, of a phthisical family, had usually enjoyed good, though not strong, health. The chief points which had been noticed about her, were the great amount of sleep which her constitution seemed to require, and a liability to uterine hæmorrhage on occasion of any excitement, however trivial. A few weeks before I saw her, she had fallen into the water, but no harm nor cold resulted. A fortnight before I saw her, she had gone to stay with some friends and during her visit, she became suddenly quite prostrated from no obvious cause. She was brought home with an expressed suspicion that she had phthisis, and had ever since been confiried to her room.

The excessive alarm of her friends was not justified by a more particular examination of the patient; for the bodily functions were all proceeding quite regularly, and the pulse did not exceed 70 in the minute. On auscultation, the respiratory murmur was generally feeble, and the expiration in the apex of the right lung above the clavicle was prolonged. There were no moist sounds; but there was tenderness, and perhaps some dulness, on percussion, beneath the right clavicle. These were the only deviations from the normal condition then discoverable.

She was put on the use of ferro-citrate of quinine, which at first made her feverish, but afterwards was tolerated. After about six weeks, I saw her again. She was very pale, but not thinner; still so very weak, that she had been confined to the sofa all the time. Auscultation still gave the same very equivocal results. The respiratory murmur was feeble in both suprascapular regions, and the resonance on pereussion was less on the right than on the left side. Beneath the right clavicle, the expiration was distinctly prolonged; and here there was imperfect pectoriloquy. But there was no dulness nor tenderness on percussion here now ; still no moist sounds anywhere.

After about ten months, I saw her again. She had taken cod-liver oil with benefit all that winter, and had continued seemingly well all the following summer. With the coming autumn she appeared to be failing again. Auscultation revealed nothing more distinct. But there was no question that the right suprascapular region was duller to percussion than the left. Thus far the disease had advanced, and then stopped.

And still after eight years she goes on leading a half-invalid life, inactive by nature, and apparently incapable of exertion from want of strength. The disease has made no further progress in the apex of the right lung.

What is the further progress of the cases of which this is a type? To my own experience, it is much as follows:-

Many of these cases stand still, like that just detailed. The disease stops within narrow limits, however great within those limits the morbid changes may be. The patients remain weakly and delicate, though perhaps not more so than they were before; and the physical signs remain the same; whispering or exaggerated vocal resonance, with some dulness on percussion, over the affected part; and perhaps now and then a few moist sounds. Such a condition is not reparation in any proper sense of the word. It is only arrest of the disease, a condition of comfort indeed more or less complete while it lasts; but liable to be interrupted by accidents from within or from without.

Others of them, however, do get thoroughly well; and pathology is slowly accumulating the pronf of cicatrisation of tubercular cavities, and investigating the circumstances under which this favourable issue may be expected.

It is in these chronic cases limited to a small portion of the lung that treatment does so much good; for there is time to observe the character of the disease in the particular case; and the result of an error in the first instarice, if so it should be, as in the choice of a climate or the plan of treatment, are not irremediable. Nature is doing everythin $y$ to sepair the foregone mischief; and though we may do little to help this, we may do much to keep off any noxious influences. 'The earlier the disease comes under observation and treatment, the more complete is the cure likely to be. It is not so many months added to life by daily care; it is, so to say, life itself.

This hopeful view of the occasional results of limited tubercular deposit, contrasts strongly with the condition illustrated in the following case.

CaSE Iv. Definite Outbreak of Diffuse Tubercle. Emma Green, aged 23, was admitted under my care into the Sussex County Hospital, on October 1st, 1854. She was healthy looking, but hurried and nervous; a nursemaid, who had enjoyed good health till the previous winter, when she caught a sligh cold, which had hung about her ever since. 'Three weeks before admission, she was suddenly seized with a sharp pain in the right side of the chest, which had continued ever since and, on admission, constituted her chief complaint.

On examination of the chest, the left side was found quite normal. On the right side, behind, there were healthy respiration and resonance on percussion in the upper half, feeble but otherwise healthy respiration in the lower half, with scanty small crepitation on deep inspiration, and dulness on percussion. In front, nothing deviating from the condition of health could be detected. It seemed probable that she had pleurisy with slight effusion on the right side.

The notes of the next four weeks only speak of the gradual removal of the fluid from the pleura, under the use of blisters, and of a very cautious employment of mercury and antimony. But they constantly speak of a hurry of the pulse, more than mere passing nervousness would be likely to induce, and of a very loaded state of the urine. On October 27 th, on occasion of a complaint of slight pain in the front of the chest, this part was carefully examined. There was then loud puerile breathing beneath the left clavicle. Beneath the right clavicle, the inspiration was feeble, and followed by a few small sharp crepitations. Expiration was prolonged; and it was thought that the resonance on percussion was slightly impaired. On this point, however, at this period, some doubts were expressed. The notes of the next six weeks speak of continually in- 
creasing weakness, nausea, anorexia, and fever, with distressing cough, but no expectoration: and the successive recorded auscultations describe a rapid extension of the physical signs of discase of the upper part of the right lung. These are detailed in the notes of December 1st and 15th, as abundant moist sounds throughout the upper half of the right lung behind; in front, all over the right side, coarse cavernous breathing, with prolonged expiration and abundant mixed crepitation at the turn of respiration, with great dulness on percussion.

About this time, advantage was taken of an improvement in her general symptoms to remove her to her home in a distant part of the country. The improvement seemed at the time to be probably one of those brief respites which we so often meet with in the course of phthisis. But it proved otherwise. She survived for nearly six months after leaving the hospital.

This case needs little more comment than to anticipate the inference which may have already suggested itself, that probably it was a case of tubercular disease throughout. Probably (in the absence of anatomical demonstration) the disease changed its seat only, not its nature, when the pleural were exchanged for pulmonary symptoms.

These are two extreme cases, so dissimilar in their whole progress from the beginning throughout, that they do not appear to have had in common, besides the auscultatory evidence, any very important characteristic of the common disease. The rapid pulse and fever in one case contrast strongly with the mere languid debility in the other. I would dwell on their dissimilarity as enforcing the pathological importance of the anatomical distinction with which it was apparently connected.

But that this distinction may not engross too much time, I would proceed to another part of the subject. Such extreme cases as those which have been detailed above, like all other extremes, are proportionately rare: the mass is made up of cases which are only to be adjusted to any particular scale indistinctly and with difficulty. Yet, among the countless forms of the disease which defy any systematic arrangement, some recur with sufficient frequency to allow of the cases being grouped into a kind of natural order. Not to attach too much importance to any such classification, at least a useful hint may often be obtained in a perplexing case, by its resemblance to other cases constituting a little group of this kind.

Acute phthisis constitutes such a natural group, characterised by its own very distinct symptoms. The negative symptoms are much more characteristic than the positive; yet, once or twice seen, the disease is not difficult to be recognised. There is the fever, and much of the general appear. ance of pneumonia ; but the physical signs of pneumonia are wanting. Such abnormal sourids as the ear can detect, few as they are, seem generally referrible to bronchitis. But the amount of bronchitis which they indicate is far too small to explain all the other symptoms. This doubt, however-this very obscurity-is a most important element in the diagnosis of acute phthisis.

A dusky face, a hurried pulse, loaded urine, and other indications of irritative fever, accompanied by singular prostration of strength-these are the conditions under which acute tuberculosis of the lungs commonly presents itself to notice. Auscultation finds almost nothing abnormal. There may be no cough; commonly there is no expectoration; and there are few or no moist sounds. There may be absolutely nothing to notice but slight general impairment of the respiratory murmur, often without even any prolongation of the expiration; and there is no perceptible want of resonance on percussion.

Much of this obscurity is inseparable from the nature of the case. For the affection being general throughout both lungs, we cannot learn anything from a comparison of the two sides, or of different parts of the same side of the chest. In this condition some of these patients die, and dissection shows their lungs to be filled with miliary tubercles. These are not yet voluminous enough to affect the normal degree of resonance on percussion; or rather, as I believe, this effect of their presence is neutralised by a slightly emphysematous condition of the lungs generally. But that they do really, directly or indirectly, obstruct the functions of the lungs, is proved by the feeble respiratory murmur, coinciding with the dusky surface of the body.

It is rarely, however, that the subjects of acute phthisis sink thus early. As life is prolonged, the signs and symptoms of pulmonary disease are expressed less equivocally. But to the condition of the lungs which now ensues it would be very difficult to apply any very exact name, either from auscultation during life or from anatomical examination after death. Many causes are in active operation, and it is hard to disentangle their combined results. In a few words, dissection displays the results of the softening of tubercles scattered throughout the whole of the lungs, combined with inflammation of whatever structures of the lungs or of its investments they may occupy.

The more protracted cases are gradually cleared up as time goes on, when the ordinary physical signs, and the tendency of the disease to settle on particular points, manifest themselves. But in the very rapid cases, where the seeming duration of the disease, from its beginning to its fatal termination, is to be counted by days rather than by weeks, the diagnosis rests on no positive signs. And for the most part, at first sight, pneumonia, bronchitis, or continued fever, offer themselves as the hopeful alternatives of acute phthisis.

Obscure as they are, yet, taken as a whole, these cases will be found to have a tolerably uniform history of more or less prolonged ailing, on which the symptoms of acute disease have at last suddenly engrafted themselves. A review of this period generally favours the conclusion that the pulmonary tuberculosis is only the local expression of the constitutional disease, and that the symptoms and changes to which we apply the terms of acute phthisis are only the overt development of the latent tendency, the bursting of the clouds which had been silently gathering for a long while before.

It would be most desirable to have some insight into what is going on during this period, when, if ever, we may hope to be able to interfere with our remedies to any purpose. But our powers of interpreting the signs of the coming disease must be confessed to be very limited, and the grounds of our predictions very uncertain.

These cases, where we are compelled to hold our judgment in suspense, are well adapted to test the value of the specific signs of disease which we do not need, and so often disparage when we have better, but which may yet often give a useful hint in the absence of more certain information. Such hints may be drawn from the ungues adunci and the facies phthisicorum amabilis of the older authors, as well as from the red edges of the gums to which later writers have directed attention. And there is another symptom recently noticed by $\mathrm{Dr}$. Seymour, namely, constant vomiting, the value of which I would attest. It is not a common symptom; indeed, characteristic cases of this kind are, to my own experience, very rare.* The explanation of it is probably to be found in the physiological relations of the digestive and respiratory branches of the eighth pair of nerves, relations whose study has been prolific of such important results in the hands of M. Claude Bernard. But whether or not this symptom of continued vomiting can claim a high pathological interest on these grounds, still, for other reasons, it is worth careful attention in suspected cases of phthisis. For it characterises a form of the disease which, once developed in the lungs, runs rapidly to a fatal termination. And it seems closely connected with a certain stage only of phthisis; for, with the manifestation of the rapid succession of changes which the softening tubercle induces in the lungs, the vomiting commonly ceases.

The treatment of phthisis is altogether beyond the scope of the present remarks. All I would venture to say of what should be done during the anxious period when we are waiting to see whether phthisis will develope itself, is contained in the wise

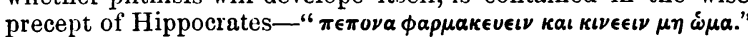
Phthisis is, indeed, a disease of debility; but it is not to be prevented at this, any more than it is to be cured at any subsequent period, by tonic or stimulant treatment. On the contrary, the symptoms for the relief of which we are most often compelled to interfere, and over which we have most influence at the commencement of acute phthisis, are commonly inflammatory. And the exhaustion consequent on the cautious use of antiphlogistic measures is incomparably less than that which would be induced by the continuance of active disease.

In a prospective point of view, acute phthisis commands great attention. But, with its manifestation, its pathological, or at least its clinical interest abates. For, once developed, it is not often in our power to arrest, or materially to interfere with its progress. The limits, moreover, within which we may profitably subdivide the varieties of acute phthisis are very narrow, for acute phthisis is itself but a single form of consumptive disease. It is among chronic cases, as their longer duration allows, that we find the more numerous and more striking varieties.

The following is not an uncommon case:-A person in active

* Vomiting in this form should be distinguished from that which appears to depend on agitation of the stomach by cough, or on nausea arising from any pulmonary excretions which may have been swallowed. 
and seemingly strong health is suddenly seized with an acute inflammatory attack of an uncertain nature. There may be just cough or pain enough to direct attention to the chest; where, perhaps, we may find the physical signs of slight bronchitis verging into pneumonia. And these physical signs may continue for many days or even weeks unchanged. As they change, at last, the physical signs of tuberculisation of the lungs become manifest. And now the nature of the case is clear.

Some of these cases run on rapidly as acute phthisis, but others assume a very peculiar form. My experience of these is not sufficiently large to say in what way these terminate, or how long their end may be deferred; whether their duration be a question of months or years; a lengthening acquaintance with some of these would, however, lead me to assign them the longer term of duration. But their characters as a group are worthy of attention. One day, as it were, the patient was strong and active; perhaps more active than really strong. But, dating from that day, henceforth he is an invalid. And for the continuance of that invalid existence, he is bound to a most scrupulons observance of the rules which his own painful experience inculcates. It is an experience very soon acquired. For a neglect of its rules is as surely as speedily attended by a painful feverish attack, followed by long continued weakness. And the recovery from each such attack is always to a point lower in the scale of useful health than that which he occupied before.

Perhaps the most striking characteristic of these cases is the freedom from the usual symptoms of phthisis in the intervals of the attacks. The patient lives on the verge of these symptoms, which the slightest indiscretion will call'into existence. But keeping within certain limits he may almost entirely escape them. This immunity, however, is less complete as the case goes on, and as the growing amount of organic disease of the lung renders the organ more susceptible of any injurious influences.

As to the nature of the inflammatory attack with which this form of consumption begins, I believe the disease to be essentislly, though not exclusively, tubercular, from the beginning. The tubercle does not seem to be added to the original deposit; but we detect its proper physical signs as the other signs clear up. It is not an entire change, but a clearing up, which brings them to our notice. It is as if the ordinary products of inflammation and tubercle had been intermingled, and had contemporaneously gone through their respective changes, the simple disease to resolution, the tubercle to softening and erosion. And, as the curable disease passes away, the incurable comes fully into view. As the dulness on percussion becomes less extended, within those narrowing limits it becomes more intense. The fading bronchial sounds are gradually replaced by the signs of erosion of the lung.

We may regard these cases in their beginning, I believe, as tuberculosis limited to a portion of the lung, but attended by an unusual amount of local irritation. This view, however, can be maintained only on general grounds in preference to that which would regard the tubercular deposit as the consequence, not the cause, of the inflammation.

I have observed a very singular form of phthisis, where the symptoms of hectic fever have been manifested very early and with so much regularity, as to assume the type of tertian or quotidian ague. The three cases wnich I have particularly in mind at present had little in common, save that they were all women and hospital patients, and that their most prominent complaint was the intermittent paroxysm of fever. In one of them the paroxysms gradually assumed the regular tertian type while she was in the hospital. The effect of quinine in stopping their recurrence was here as distinct and rapid as in ague. And, indeed, I cannot wholly reject the opinion that she might have had ague.

Apart from the question of diagnosis, I would not lay any particular stress on this form of phthisis. For its very rarity, one chief cause of my putting it forward here, detracts from its general importance. The difference of treatment involved, according to the different reasonable suppositions of the cause of the symptoms, is not great, nor the alternatives perilous And the indications are perfectly obvious throughout. Only one word more:-Rare as this form of disease has appeared to be, I should have been much surprised never to have noticed such a conjunction of symptoms. It would have been matter of wonder, had irritation of the lungs by organic disease of such a nature not found that form of constitutional sympathy which is excited by abscess of the brain, or by valvular disease of the heart.

[To be continued.]

\section{OBSTINATE HAMORRHAGE FOLLOWING ABORTION : RECOVERY.}

By R. Jones, Esq., Strefford, Shrewsbury.

ThE perusal of Mr. Humphreys' Case of Uterine Hæmorrhage, in the Jounnal for December 4 th, induces me to relate briefly a very similar case, which occurred in my practice some years ago. The subject of it was the wife of a blacksmith residing near this village, over 30 years of age. She was a woman of good constitution; but the tone of her system had been considerably reduced by privation and anxiety, induced by the prolonged and serious illness of her husband. She had hardly completed the third month of utero-gestation, when she experienced a sudden fright, and abortion was the consequence. The ovum was reported to have been expelled; and nothing alarming happened till some days afterwards, when she suddenly jumped out of bed to assist her husband in an emergency. Profuse loss followed; and $I$ found her in a fainting state, with copious gushes escaping every two or three minutes. A plug applied put a stop to this at once. For the next month or five weeks, the same recurred, in spite of all treatment suggested, including the mineral acids, metallic salts, vegetable styptics, opium, and ergot, with cold affusions and astringent voginal injections. On many occasions, the timely use of the plug appeared to rescue her from impending dissolution: all other treatment was of no avail; and such was the fearful condition to which my patient was reduced, that $I$ looked upon her case as all but hopeless. As a dernier ressort, I decided to try the effect of mercury. One grain of calomel and half a grain of opium was, therefore, given every six hours. The bowels were relieved occasionally by cold water injections, and active counterirritation was applied to the sacrum. No serious hæmorrhage happened afterwards, and my patient made a slow but perfect recovery.

I have adopted the same treatment, with the same success, in several obstinate cases of the same character since; but of late years I have placed much confidence in another remedy for such cases, and have seldom been disappointed by it. That remedy is oil of turpentine.

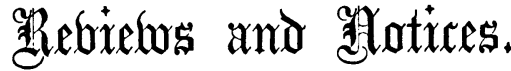

On Chloroform and other Anesthetics: thetr ACtion and Administration. By John Snow, M.D., Licentiate of the Royal College of Physicians. Edited, with a Memoir of the Author, by Benjanis W. Richardson, M.D., Licentiate of the Royal College of Physicians. Pp. 443. London: John Churchill. 1858.

IN undertaking to review Dr. SNow's book, it is necessary to do so as if the writer were still alive, although that course may appear somewhat ungracious, and is, no doubt, not perfectly just, since any defects which now appear in it might, and very probably would, have been corrected, had the author lived to revise it. He seems, however, to have left no notes or manuscript to guide in the continuation of the work bejond what now appears as the last sentence, during the writing of which he was seized with the apoplexy which proved fatal to him. The task of editing it devolved naturally on Dr. Richurdson, as the intimate friend of the deceased; and he has prefixed to the book a Memoir, which is entertaining, and not uninstructive, as exhibiting the struggles of a poor man of sterling integrity and much merit, but destitute of those popular talents by which early success is sometimes attained.

Of Dr. Snow's work we have no difficulty in expressing a very high opinion. In it an important subject is treated fully, and in the only way in which such a subject ought to be treated; vi६. by a combination of experience, reasoning, and experiments. Yet the work has some grave omissions and defects; and, as its merits are so conspicuous, we need have the less hesitation in pointing them out. The most striking omissions-to the probable cause of which we have already referred -are the utter silence of the author on the very important subject of local anæsthesia, and on the supposed effects of the admi nistration of chloroform upon the healing process. We must 